\title{
Pancreatic Resections after Chemoradiotherapy for Locally Advanced Ductal Adenocarcinoma: Analysis of Perioperative Outcome and Survival
}

\author{
Paolo Massucco, MD,${ }^{1}$ Lorenzo Capussotti, MD, ${ }^{1}$ Antonella Magnino, $\mathrm{MD},{ }^{2}$ \\ Elisa Sperti, MD ${ }^{2}$ Marco Gatti, MD,${ }^{3}$ Andrea Muratore, MD,${ }^{1}$ Enrico Sgotto, $\mathrm{MD},{ }^{1}$ \\ Pietro Gabriele, $\mathrm{MD}^{3}$ and Massimo Aglietta, $\mathrm{MD}^{2}$
}

\footnotetext{
${ }^{1}$ Unit of Surgical Oncology, Institute for Research and Cure of Cancer, 10060 Candiolo, Italy

${ }^{2}$ Unit of Medical Oncology, Institute for Research and Cure of Cancer, 10060 Candiolo, Italy

${ }^{3}$ Unit of Radiation Therapy, Institute for Research and Cure of Cancer, 10060 Candiolo, Italy
}

\begin{abstract}
Background: The most accepted treatment for locally advanced pancreatic cancer is chemoradiotherapy. However, indications to and results of pancreatic resections after chemoradiation are not yet defined.

Methods: From June 1999 to December 2003, 28 patients with locally advanced pancreatic cancer (group 1) were enrolled for institutional trials of gemcitabine-based chemoradiotherapy. Tumors were stratified as unresectable or borderline resectable according to the pattern of vascular involvement at pretreatment computed tomographic scan. Patients with partial response or stable disease and in-range Ca19-9 were surgically explored. Perioperative outcome and survival of group 1 were compared with 44 patients primary resected for localized cancer with or without adjuvant treatment in the same time period (group 2).

Results: Only one unresectable tumor was successfully resected compared to 7 out of 18 (39\%) that were borderline resectable. Operations after chemoradiation were 1 hour longer and postoperative stays 5 days longer, but transfusion rate, morbidity, and mortality were not significantly different. Median survival was 15.4 months for group 1 ( $>21$ for resected vs. 10 for not resected, $P<0.01$ ) and 14 months for group 2. In both groups, a disease-free survival beyond 24 months was recorded only among patients resected with negative margins.

Conclusions: The conversion of an unresectable cancer to a resectable one is a rare event. On the contrary, the resection of a borderline resectable tumor was successfully accomplished in one-third of cases. Chemoradiotherapy did not increase the operative risk, but the interventions were more technically demanding and required a longer postoperative stay. Patients resected after chemoradiation for a locally advanced tumor had at least the same survival as those primary resected for a localized one. Only R0 resections in both groups gave the chance of disease-free survival longer than 24 months.
\end{abstract}

Key Words: Pancreatic neoplasm - Combined modality therapy — Surgery - Locally advanced cancer.

Pancreatic cancer continues to be the most lethal gastrointestinal neoplasm owing to the advanced

Received October 17, 2005; accepted April 05, 2006; published online September 6, 2006.

Address correspondence and reprint requests to: Paolo Massucco, MD; E-mail: pmassucco@mauriziano.it

Published by Springer Science+Business Media, Inc. ㄷ 2006 The Society of Surgical Oncology, Inc. stage at diagnosis and the lack of effective treatment. Pancreatic resection is the only potentially curative therapy, but unfortunately, fewer than $20 \%$ of cases are amenable to operation at presentation. Up to $40 \%$ of all cases have no evidence of distant metastases but are borderline resectable or unresectable because of the local spread of disease (locally advanced neoplasm). On the basis of a few randomized 
trials, the most accepted treatment for this condition is chemoradiotherapy. ${ }^{1-4}$ In the last decade, many regimens have been proposed with encouraging results. $^{5-18}$ However, the indications, if any, and the results of pancreatic resections after chemoradiotherapy are not yet clear.

Since 1999, patients presenting at our institution with a locally advanced pancreatic adenocarcinoma were enrolled in a phase II chemoradiation treatment protocol. The results of this study have been reported elsewere. ${ }^{19}$ In this report, we present the perioperative and long-term outcomes of patients resected after chemoradiation, comparing them with primary resections consecutively performed during the same time period.

\section{PATIENTS AND METHODS}

\section{Patient Population and Treatments}

From June 1999 to December 2003, 28 patients with locally advanced pancreatic cancer (group 1) were enrolled for institutional phase II trials of chemoradiotherapy. In all cases, cytological diagnosis of ductal adenocarcinoma was obtained by computed tomography (CT)-guided fine needle aspiration. Determination of local and distant spread was based on a thin-section enhanced multidetector CT scan, with vascular reconstruction when needed. Patients with extrapancreatic disease or with massive retroperitoneal nodal involvement (i.e., outside the resection field) were not eligible. The tumor was defined as locally advanced in the presence of (1) abutment (lack of a fat plane between the tumor and the arterial wall) or encasement of celiac axis (CA) or superior mesenteric artery (SMA) (cT4) or (2) involvement of superior mesenteric-portal vein axis with either stenosis or thrombosis of the vessel $(\mathrm{cT} 3 \mathrm{v}+)$. Patients were then stratified as (1) unresectable (in the presence of vein thrombosis and/or arterial encasement) or (2) borderline resectable (in the presence of vein stenosis and/or arterial abutment).

Ca19.9 serum level was recorded at registration. The treatment protocol of the first 23 patients has been previously detailed. ${ }^{19}$ In brief, patients received three-dimensional conformal radiotherapy with highenergy X-rays; the clinical target volume (CTV) included the entire lesion and the nodal areas at risk for a total dose of 45 Gy (1.8 Gy/day). Concurrent chemotherapy consisted of gemcitabine administered as a 60-minute intravenous infusion each Monday and Thursday for 5 weeks. Gemcitabine was given within 2 hours before radiation treatment at a dose of $100 \mathrm{mg} /$ $\mathrm{m}^{2}$ twice weekly in the first 15 patients. The dose was reduced to $50 \mathrm{mg} / \mathrm{m}^{2}$ twice weekly in the subsequent eight patients because of the toxicity observed and the difficulty in giving the planned dose. The last five patients were enrolled in a novel institutional protocol and received two courses of induction gemcitabineand oxaliplatin-based chemotherapy before chemoradiation. Eligible patients had to sign an informed consent form before enrollment into the trials.

\section{Response Assessment and Indication for Operation}

The therapeutic response was evaluated according to responce evaluation criteria in solid tumours (RECIST) criteria by a CT scan performed 6-8 weeks after the end of treatment. Surgical exploration was scheduled in patients who experienced downsizing of the mass or had a stable disease with in-range Ca19-9. In the absence of distant progression at laparotomy, a pancreatic resection (Whipple procedure or distal splenopancreatectomy) with extended lymphadenectomy was performed as previously described. ${ }^{20}$ In summary, the lymphadenectomy field included the nodal groups of hepatic pedicle, hepatic artery, CA, SMA on its right and anterior aspect, and the preaortic retroperitoneal tissue between the CA and inferior mesenteric artery. A total pancreatectomy was performed if a free pancreatic resection margin was not obtained from intraoperative frozen section examination. When an adhesion between the tumor and portal/mesenteric vein wall precluding the identification of a clear cleavage plane was encountered during the dissection, a vein resection was performed without any attempt to directly document the supposed vein infiltration during the operation. Pathological stage was defined according to the International Union against Cancer (UICC) classification system. ${ }^{21}$ Resection margins, including the retroperitoneal and uncinate surfaces, were checked for neoplastic invasion. Nodal metastases were classified as regional or extraregional if found in the preaortic nodal group.

\section{Analysis of Results}

The perioperative and long-term outcomes of group 1 patients were compared with a group of 44 patients who in the same period underwent a primary resection for localized ductal adenocarcinoma with or without adjuvant gemcitabine-based chemotherapy or chemoradiation (group 2). The adjuvant therapy has been given since the year 2002 in patients who 
were able to start the treatment within 2 months of operation. Mortality, morbidity, operative time, transfusion rate, and hospital stay were recorded. All patients were followed by abdominal ultrasound and CA19-9 dosage every 4 months for the first 2 years and every 6 months thereafter. A thoracoabdominal CT scan was performed yearly. Additional tests (i.e., cytology on ascites or pleural effusion) were performed when a recurrence was suspected. Data were prospectively collected on a dedicated database. Survival was computed from the date of treatment initiation, i.e., the starting date of medical treatment for group 1 and the date of operation for group 2 . The site of recurrence was recorded as local (any mass in the resection field), peritoneal, or distant. Followup was updated at June 2005.

Continuous variables were expressed as mean \pm standard deviation and compared with a two-tailed $t$-test. Categoric variables were compared with K-square or Fisher's exact test as appropriate. Survival curves were estimated with the KaplanMeier method and compared by the log-rank test. Overall survival was computed considering as events all the deaths whatever the cause, including postoperative deaths. The events considered for disease-free survival computation were all deaths and all recurrences. An analysis of prognostic factors for survival was performed including all resected patients from groups 1 and 2 and considering as covariates sex, age $>70$ years, type of operation (partial vs. total pancreatectomy), perioperative transfusions, grading (G1-2 vs. G3), T stage (T1-2 vs. T3), N stage (positive vs. negative), presence of neoplastic emboli, presence of perineural invasion, $\mathrm{R}$ status, neoadjuvant therapy (resection after neoadjuvant therapy vs. primary resection), and any form of chemotherapy (neoadjuvant or adjuvant therapy vs. primary resection alone). $P<0.05$ was considered significant in all cases.

\section{RESULTS}

Patients' characteristics are summarized in Table 1. Group 1 patients were significantly younger but with a mean age difference of less than 5 years. Group 2 tumors had a significantly smaller diameter at staging CT scan, none had cT3v + or cT4 vascular involvement, and there was a trend toward a lower mean Ca19.9 level at presentation.

All group 1 tumors had a cT3v + or cT4 vascular involvement. Isolated vein involvement was found in five cases (four stenosis, one thrombosis). Of the 23 patients with arterial involvement, a vessel
TABLE 1. Comparison of groups

\begin{tabular}{|c|c|c|c|}
\hline & $\begin{array}{l}\text { Group } 1 \\
\text { (28 patients) }\end{array}$ & $\begin{array}{l}\text { Group } 2 \\
\text { (44 patients) }\end{array}$ & $P$ \\
\hline Sex & $12 \mathrm{~F} / 16 \mathrm{M}$ & $24 \mathrm{~F} / 20 \mathrm{M}$ & n.s. \\
\hline Age (years) & $\begin{array}{c}62.7 \pm 5.6 \\
(49-75)\end{array}$ & $\begin{array}{c}66.5 \pm 8.7 \\
(49-86)\end{array}$ & 0.05 \\
\hline Site & $\begin{array}{l}23 \mathrm{head}, \\
5 \mathrm{body} / \text { tail }\end{array}$ & $\begin{array}{l}41 \text { head, } \\
3 \text { body/tail }\end{array}$ & n.s. \\
\hline Size $(\mathrm{cm})$ & $\begin{array}{l}4.3 \pm 1.3 \\
(2.8-8)\end{array}$ & $\begin{array}{c}2.2 \pm 0.6 \\
(1-3)\end{array}$ & $<0.01$ \\
\hline \multicolumn{4}{|l|}{$\begin{array}{l}\text { Vascular } \\
\text { involvement }\end{array}$} \\
\hline cT3v + only & 5 & & \\
\hline cT4 & 23 & & \\
\hline \multicolumn{4}{|l|}{ Ca19.9 } \\
\hline Increased & $93 \%$ & $82.5 \%$ & n.s. \\
\hline Mean & $\begin{array}{c}1,053 \pm 2,869 \\
\quad(2-15,455)\end{array}$ & $\begin{array}{c}742 \pm 1,510 \\
(2-8,804)\end{array}$ & n.s. \\
\hline
\end{tabular}

Data are mean \pm standard deviation and range, unless otherwise noted.

encasement was evident in eight (SMA in two, CT in four, and both in two) and the absence of a fat plane with the vessel wall in 15 (SMA in three, CT in six, and both in six). In all but three of these 23 patients, vein involvement was present as well. In five patients, preaortic enlarged lymph nodes were detected in the field of the planned lymphadenectomy (between CT and inferior mesenteric artery). As for resectability status, 10 patients were unresectable (three for vein thrombosis and seven for arterial encasement) and 18 were borderline resectable, four of whom had isolated vein stenosis.

\section{Toxicity and Response}

All 28 group 1 patients were assessable for toxicity. A detailed report of toxicities registered during our first institutional protocol has been previously reported. ${ }^{19}$ In summary, at the gemcitabine dose of 100 $\mathrm{mg} / \mathrm{m}^{2}$, three of the 15 patients had to stop the treatment because of toxicities $>$ G3. One patient needed hospitalization for gastrointestinal toxicity, and three required a temporary interruption because of hematological and gastrointestinal toxicity. The median dose received was $65 \%$ of the planned dose. At the $50 \mathrm{mg} / \mathrm{m}^{2}$ dose, all patients completed the treatment; none needed hospitalization. The median dose received was $80 \%$ of the planned dose; no one needed dose reduction. Among the five patients treated with the new protocol, all completed the induction chemotherapy course but two required interruption of chemotherapy during radiation. No deaths or life-threatening toxic reactions were directly attributable to chemoradiation.

Of the 23 patients who were assessable for response, we observed a partial response in five (22\%), 
stable disease in $14(61 \%)$, and disease progression in four $(17 \%)$. All partial responders had a borderline resectable tumor at presentation. Among the four patients who had disease progression, three developed distant metastases and one, a portal thrombosis. The latter was the only case of evolution from borderline resectability to unresectability because of local progression. A reduction of serum Ca19.9 was observed in 15 of $21(71 \%)$ patients with an elevated level at presentation. Normalization (complete marker response) was evidenced in five patients, three with partial response and two with stable disease.

Eight patients (29\%) were scheduled for operation, five with a partial response and three with stable disease but in-range Ca19.9 at restaging (two for normalization and one in-range at presentation). All patients explored were successfully resected. Considering the pretreatment resectability status, seven of the 18 (39\%) borderline resectable cases underwent a pancreatic resection, including all four cases with isolated vein stenosis. Only one unresectable patient (for a limited vein thrombosis) was brought to resection. Of the 11 borderline resectable patients who did not undergo operation, one died of sepsis related to hepatic abscesses before surgical evaluation, four were not available for response, four had disease progression, and two had stable disease but were not explored because of stomach infiltration in one case and increasing serum marker in a head neoplasm with adhesion to the CA, SMA, and portal vein in the other.

\section{Surgery and Pathology}

The operations performed for group 1 patients were four duodenopancreatectomies and four total pancreatectomies. In three cases, a segmental resection of the superior mesenteric vein was necessary. One patient died 2 months after operation due to failure to thrive after a biliary leak treated radiologically. Complications were registered in four cases: one biliary leak, one pancreatic fistula, and two delayed gastric emptying. No reoperations were necessary.

In the same period, group 2 patients underwent 28 pancreatoduodenectomies, 13 total pancreatectomies, and three distal splenopancreatectomies. Resection of the mesenteric vein confluence was needed in one patient. Two patients died in the postoperative period. One patient was reexplored for hemoperitoneum associated to a pancreatic fistula and died of septic shock. Another patient died of systemic candidiasis without evidence of abdominal complications.
TABLE 2. Perioperative outcome

\begin{tabular}{llll}
\hline & $\begin{array}{l}\text { Group 1 resected } \\
\text { (8 patients) }\end{array}$ & $\begin{array}{l}\text { Group 2 } \\
\text { (41 patients) }\end{array}$ & $P$ \\
\hline Operation type & $\begin{array}{c}4 \text { Whipple, } \\
\text { 4 total }\end{array}$ & 28 Whipple, 13 total & n.s. \\
$\begin{array}{c}\text { Vein resection } \\
3 / 8\end{array}$ & $1 / 41$ & $<0.01$ \\
$\begin{array}{c}\text { Operative time } \\
\text { (mean, hours) }\end{array}$ & $6.6 \pm 0.5$ & $5.5 \pm 1.0$ & $<0.01$ \\
$\begin{array}{c}\text { Transfusion rate } \\
\text { Hospital stay } \\
\text { (median, days) }\end{array}$ & $1 / 8$ & $9 / 41$ & n.s. \\
$\begin{array}{c}\text { Mortality } \\
\text { Morbidity }\end{array}$ & $1 / 8$ & 13.5 & 0.02 \\
\hline
\end{tabular}

Morbidity was 36\% (16/44). The more frequent complications were pancreatic fistula (five cases) and delayed gastric emptying (three cases). Reoperations were performed in four patients: for pancreatic fistula in two cases, leak from gastroenteric anastomosis in one, and segmental colonic necrosis in one.

The analysis of perioperative outcome was performed excluding the three distal pancreatectomies from group 2, leaving 41 patients with a head cancer for comparison with group 1 resected cases. Mortality, morbidity, and transfusion rate were not significantly different between the two groups. However, operative time was 1 hour longer and hospital stay was 5 days longer for group 2. This comparison is reported in Table 2.

Final pathology of group 1 patients revealed a partial pathological response (residual microscopic cancer foci) in two cases and pT3 ductal adenocarcinoma in six cases. No one had a complete pathological response. Regional nodal involvement was found in two patients (one and two positive nodes, respectively). Among the patients with enlarged preaortic nodes at presentation, two were resected and were node-negative at final pathology. No one had extraregional nodal involvement. The retroperitoneal margin was positive in one case.

Group 2 patients had a significantly higher nodepositivity rate. The mean number of positive nodes was 4.5 . Clinically unsuspected extraregional positive nodes (stage IV) were found in five cases. There was a trend toward a higher rate of retroperitoneal margin positivity. The pathological features are presented in Table 3.

\section{Survival}

Median overall survival and disease-free survival were 15.4 and 10 months for group 1 and 14 and 8 months for group 2, respectively. Group 1 patients who underwent resection had significantly longer 
TABLE 3. Pathological findings

\begin{tabular}{lccc}
\hline & $\begin{array}{l}\text { Group 1 resected } \\
\text { (8 patients) }\end{array}$ & $\begin{array}{l}\text { Group 2 } \\
\text { (44 patients) }\end{array}$ & $P$ \\
\hline G3 & $3 / 7$ & $18 / 43(58.1 \%)$ & n.s. \\
$\mathrm{N}+$ & $2 / 8$ & $36 / 44(81.8 \%)$ & $<0.01$ \\
T1-2 & $1 / 8$ & $6 / 44(14 \%)$ & n.s. \\
T3 & $7 / 8$ & $38 / 44(86 \%)$ & \\
pTNM stage & & & \\
IIa & $6 / 8$ & $8 / 44(18 \%)$ & $<0.01$ \\
IIb & $2 / 8$ & $31 / 44(70 \%)$ & \\
IV & & $5 / 44(11 \%)$ & \\
Neoplastic emboli & $5 / 6$ & $30 / 33(91 \%)$ & n.s. \\
Perineural invasion & $3 / 6$ & $25 / 33(76 \%)$ & n.s. \\
R + & $1 / 8$ & $14 / 44(32 \%)$ & n.s. \\
\hline
\end{tabular}

TABLE 4. Long-term outcome

\begin{tabular}{|c|c|c|c|c|}
\hline & $\begin{array}{l}\text { Median } \\
\text { OS }\end{array}$ & $\begin{array}{l}\text { 2-year } \\
\text { OS }\end{array}$ & $\begin{array}{l}\text { Median } \\
\text { DFS }\end{array}$ & $\begin{array}{l}\text { 2-year } \\
\text { DFS }\end{array}$ \\
\hline Group 1 & 15.4 & $13.4 \%$ & 10 & $7.1 \%$ \\
\hline Resection $(\mathrm{n}=8)$ & $>21$ & $52.5 \%$ & 12 & $18.8 \%$ \\
\hline $\begin{array}{l}\text { No resection } \\
(\mathrm{n}=20)\end{array}$ & 10 & $0 \%$ & $4.5^{a}$ & $0 \%$ \\
\hline Group 2 & 14 & $35.3 \%$ & 8 & $17.3 \%$ \\
\hline $\begin{array}{l}\text { Adjuvant treatment } \\
(\mathrm{n}=15)\end{array}$ & 20.6 & $49.2 \%$ & 10.3 & $17.5 \%$ \\
\hline $\begin{array}{l}\text { No adjuvant treatment } \\
(\mathrm{n}=29)\end{array}$ & 10.9 & $29 \%$ & 5.4 & $16.5 \%$ \\
\hline
\end{tabular}

${ }^{a}$ Time to progression.

OS, overall survival; DFS, disease-free survival.

overall survival and disease-free survival compared to those not resected $(P<0.01)$. This difference persisted even when only borderline resectable cases were left for comparison $(P<0.01)$. Group 2 patients who received adjuvant treatment had twofold the survival of untreated patients even if statistical significance was not reached $(P=0.17)$. The detailed survival figures are shown in Table 4. Figure 1 shows the survival curves for group 1 patients stratified as resected and not resected compared to group 2 patients.

At the time of analysis, 18 out of 20 group 1 patients who did not undergo operation died of disease within 24 months, one died of sepsis after the end of treatment, and one is alive with peritoneal progression at 14 months. Operative deaths excluded, among the seven group 1 resected patients, two died of disease, whereas five are alive, three with disease recurrence and two disease-free at 42 and 17 months. Among the 42 group 2 primary resected patients, 25 died of disease, eight are alive with disease recurrence, and nine are alive and disease-free with a median follow up of 14 months. No one with positive extraregional nodes was alive beyond 18 months.

Patterns of recurrence were similar in the two resected groups. Among group 1 resected patients,

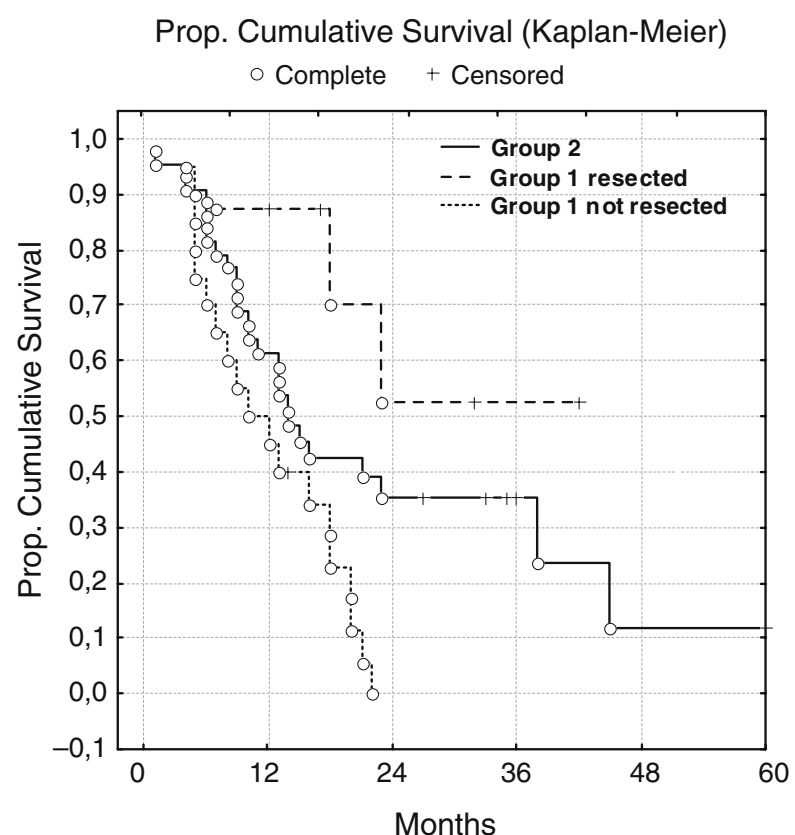

FIG. 1. Overall survival of group 1 patients, stratified as resected and not resected, compared to group 2 .

two experienced a local relapse (one isolated and one local and peritoneal), one peritoneal and two distant. In group 2 patients, the site of first recurrence was documented in 26 cases: 12 local (four isolated and eight with distant metastases), one peritoneal, and 13 distant (two with peritoneal disease).

The univariate analysis of overall survival for all group 1 and 2 resected patients identified as prognostic factors "any form of chemotherapy" (median 20.6 months for treated vs. 10.9 for untreated patients, $P=0.05$ ) and $\mathrm{R}$ status (median 22.3 months for R0 vs. 9.5 for R1, $P<0.02$ ). R status was the sole prognostic factor for disease-free survival (median 8.7 months for R0 vs. 5.5 for R1, $P=0.04)$. R status was significantly related to local recurrence rate $(67 \%$ for $\mathrm{R} 1$ patients vs. $20 \%$ for $\mathrm{R} 0$, $P<0.01)$. No one with retroperitoneal positive margins survived beyond 24 months.

\section{DISCUSSION}

At presentation, a substantial number of pancreatic cancers are already locally advanced. They are a challenging problem regarding type of treatment, relative role of chemotherapy and radiotherapy, and indications for surgical resection. Studies on chemoradiation in this clinical setting are largely heterogeneous and difficult to compare due to the lack of a 
clear definition of what is locally advanced or localized and to the different criteria applied to declare a patient resectable or unresectable. Moreover, data about the chance to render resectable an unresectable tumor are conflicting and indications for operation after chemoradiation are not standardized. A retrospective series of surgically staged unresectable tumors from Memorial Sloan Kettering Cancer Center $^{22}$ showed that it is virtually impossible to obtain enough downsizing to permit the resection of a tumor deemed unresectable at laparotomy. They could resect only one out of 87 patients treated with various chemoradiation protocols. On the other hand, a recent paper from Boston University ${ }^{23}$ reported the resection of all six patients deemed "not resectable by conventional Whipple resection" during a pretreatment laparotomy. Other centers have reported a resectability rate ranging $5-43 \% .^{5-7,9,13,15,18}$ This huge difference may be explained by many factors, such as the staging modalities adopted (surgical or radiological), inclusion criteria, and the effectiveness of different therapeutic protocols; but it revolves mainly around two issues: the definition of locally advanced cancer in each institution and the indications for surgical exploration based on radiological restaging.

The definition of the patient population is crucial for evaluating the potential role of surgery for locally advanced tumors. We applied rigorous radiological criteria to clearly define our study population. In agreement with other centers, ${ }^{24,25}$ we consider a highquality $\mathrm{CT}$ scan as an objective, reproducible, and reliable technique for pretreatment staging, identification of subsets of patients on the basis of vascular involvement, and definition of resectability status. In our opinion, a portal thrombosis or a major artery (SMA or CA) encasement should be regarded as unresectability criteria. Whereas patients showing an arterial abutment or in whom a vein resection can be anticipated from narrowing of the vein lumen should be classified as marginally resectable. In the present experience, we succeeded in resecting 39\% of borderline resectable tumors while, on the contrary, patients with truly unresectable neoplasms rarely became resectable. Our data clearly show that stratification of locally advanced neoplasms according to resectability status (truly unresectable or borderline resectable) is mandatory to evaluate the results of chemoradiation protocols and should be routinely reported.

The inclusion of four patients with an isolated vein stenosis among the locally advanced tumors was dictated by the observation that, in our previous experience, patients requiring a vein resection were less likely to receive a curative operation, due to the higher rate of positive retroperitoneal margins in comparison with patients operated without a vein resection. The same finding has been recently reported in a larger series from the M. D. Anderson Cancer Center. ${ }^{26}$ This prompted us to change our therapeutic strategy and resulted in a lower rate of vein resections in the group of primary resected patients in comparison to our previous reported series (1/41 vs. $22 / 100)$. Our current attitude is to consider for a program of resection and adjuvant treatment only patients with intrapancreatic cancers or abutting at most on the vein axis and to treat with chemoradiation more advanced stages, including patients with an isolated vein stenosis, in order to obtain a lower rate of positive margins.

The second debatable issue concerning the role of operation in locally advanced cancer is the indication for surgical exploration on the basis of the restaging CT scan. In the Memorial Sloan Kettering series, only patients showing a partial response allowing anticipation of a curative resection were referred for surgery. ${ }^{22}$ Contrarily, at Duke University, all patients without portal thrombosis or arterial encasement were explored. ${ }^{9}$ Those authors could resect many patients showing arterial abutment on the CA or SMA with a low rate of positive margins at final pathology. We adopted an intermediate policy, considering for resection not only partial responders at restaging but also cases of stable disease with normalization of serum markers as a surrogate for pathological response. In these cases, we did not find any sign of disease progression precluding resection at laparotomy.

The possibility of rendering resectable a locally advanced cancer by chemoradiation relies on the capability of downstaging the lesion and sterilizing the perivascular neoplastic tissue. According to previous reports, ${ }^{27-29}$ our data show an evident nodal downstaging in terms of percentage of node-positive cases, number of positive nodes for case, and type of node group involvement (regional vs. extraregional) when locally advanced were compared with localized tumors. This resulted in a significant lower final UICC stage for group 1 resected patients despite the more advanced clinical stage at presentation. Moreover, we observed a trend toward a higher rate of curative resections (i.e., with negative microscopic margins) after chemoradiation in comparison to primary resected cases. Although it is not yet proved if chemoradiation increases the percentage of negative retroperitoneal margins, ${ }^{30}$ this is a crucial point 
because margin status is the most powerful predictor of survival in many series, including the present one. Therefore, indications for resection should be based on the estimate of the grade of pathological response. Unfortunately, the current generation CT is unable to distinguish between neoplastic and fibrotic tissue. So, if, on the one hand, CT scan should be considered adequate to stage patients at presentation, on the other hand, it is unreliable for evaluating the response to treatment. One of our patients with a pathological partial response had stable disease on CT (data not shown). Data from Duke University show that posttreatment CT scans overestimate the unresectability status, thus precluding the chance of resection in $20 \%$ of patients. ${ }^{31}$ We clearly need new radiological tools capable of estimating the pathological response after combined modality treatment. CTpositron emission tomography or dynamic RM could give useful information in the near future. In the meantime, lacking reliable biological or radiological restaging criteria, it may be that a more aggressive policy than the one we adopted (i.e., to explore all patients without disease progression) could improve the rate of resections.

As for perioperative results, our data conflict with other published series. In 1997, Spitz et al. ${ }^{32}$ reported the postoperative results of a group of 41 localized tumors resected after chemoradiation compared with 19 primarily resected. They stated that operation after preoperative treatment was not associated with increased blood loss or hospital stay. Our experience is somewhat different. Pancreatectomy after chemoradiation shares the same difficulties of resection in the setting of chronic pancreatitis. In particular, we encountered in each case a sclerotic reaction that rendered problematic finding cleavage planes around major vessels and, moreover, having a good intraoperative determination of neoplastic spread. These findings may be due to the more advanced stage of our patients or the different protocol of treatment. Besides, even if morbidity was not substantially higher than in our previous report, patients required a longer postoperative stay because they are more fragile compared with those primarily resected and need a longer time to resume performance status allowing discharge.

Survival data were encouraging. Patients resected after chemoradiation had significantly longer survival than those not resected. Although they represent two groups of patients not directly comparable because the resected group includes less advanced tumors with better response to treatment, we think that this survival advantage can be attributed to the direct impact of resection on the natural history of the disease. In 2000, Snady et al. ${ }^{33}$ compared a group of patients treated with chemoradiation with or without resection to a group of less advanced cancers treated with resection with or without adjuvant therapy. They stated that for the first time the negative impact of a more advanced stage was reversed by changing the therapeutic strategy. This finding resulted, on the one hand, from the extraordinary survival of patients not resected after their chemoradiation protocol (median 21 months) and, on the other hand, from the suboptimal survival of their patients resected and treated with adjuvant therapy (median 16 months). In our opinion, these data should not be used to propose chemoradiation as initial treatment for every stage of pancreatic cancer and to minimize the role of surgery. We made the same type of comparison. Our data show that group 1 resected patients had at least the same prognosis as group 2 treated with adjuvant chemotherapy. On the contrary, group 2 patients who had not had adjuvant therapy had the same median survival as patients treated with chemoradiotherapy alone. The former finding could be interpreted as a confirmation of the potential role of preoperative chemoradiation in reversing the expected negative impact of a more advanced clinical stage, whereas the latter underscores the fact that surgery alone is not an adequate treatment for localized cancer. However, the most prominent considerations drawn from our data are the association of resection with improved survival and the importance of negative margins. In fact, disease-free survival beyond 24 months was possible only among patients resected in both groups. However, patients resected with positive margins had the same prognosis as patients treated with chemoradiation alone.

In conclusion, when patients were stratified according to the pattern of vascular involvement, the conversion of a truly unresectable cancer to a resectable one was a rare event with our current chemoradiotherapy regimes. On the contrary, the resection of a borderline resectable tumor after chemoradiation was accomplished in one-third of these patients with negative margins in most cases. Resections after chemoradiation in this group of patients did not increase the overall morbidity but were more technically demanding than primary resections for localized cancers, and patients required a longer postoperative stay. Patients resected after chemoradiation for a locally advanced tumor had at least the same survival as patients primarily resected for a localized cancer. However, in both groups, diseasefree survival beyond 24 months was possible only for 
patients resected with negative margins. The potential of chemoradiation to increase the rate of microscopic curative resections for borderline resectable locally advanced tumors and the criteria for deciding which patients to refer for surgical exploration need further study.

\section{REFERENCES}

1. Moertel CG, Childs DS, Reitemeier RJ, et al. Combined 5 -fluorouracil and supervoltage radiation therapy of locally unresectable gastrointestinal cancer. Lancet 1969; 2:865-7.

2. Moertel CG, Frytak S, Hahn RG, et al. Therapy of locally unresectable pancreatic carcinoma: a randomised comparison of high dose (6000 rads) radiation alone, moderate dose radiation (400 rads +5 -fluorouracil), and high dose radiation +5 fluorouracil: Gastrointestinal Tumour Study Group. Cancer 1981; 48:1705-10.

3. Klaassen DJ, MacIntyre JM, Catton GE, Engstrom PF, Moertel CG. Treatment of locally unresectable cancer of the stomach and pancreas: a randomized comparison of 5-fluorouracil alone with radiation plus concurrent and maintenance 5fluorouracil - an Eastern Cooperative Oncology Group study. J Clin Oncol 1985; 3:373-8.

4. Gastrointestinal Tumor Study GroupTreatment of locally unresectable carcinoma of the pancreas: comparison of combined-modality therapy (chemotherapy plus radiotherapy) to chemotherapy alone. J Natl Cancer Inst 1988; 80:751-5.

5. Kamthan AG, Morris JC, Dalton J, et al. Combined modality therapy for stage II and stage III pancreatic carcinoma. J Clin Oncol 1997; 15:2920-7.

6. Todd KE, Gloor B, Lane JS, Isacoff WH, Reber HA. Resection of locally advanced pancreatic cancer after downstaging with continuous-infusion 5-fluorouracil, mitomycin-C, leucovorin, and dipyridamole. J Gastrointest Surg 1998; 2:159-66.

7. Bajetta E, Di Bartolomeo M, Stani SC, et al. Chemoradiotherapy as preoperative treatment in locally advanced unresectable pancreatic cancer patients: results of a feasibility study. Int J Radiat Oncol Biol Phys 1999; 45:285-9.

8. Kornek GV, Schratter-Sehn A, Marczell A, et al. Treatment of unresectable, locally advanced pancreatic adenocarcinoma with combined radiochemotherapy with 5-fluorouracil, leucoverin and cisplatin. Br J Cancer 2000; 82:98-103.

9. White RR, Hurwitz HI, Morse MA, et al. Neoadjuvant chemoradiation for localized adenocarcinoma of the pancreas. Ann Surg Oncol 2001; 8:758-65.

10. Mehta VK, Poen JC, Ford JM, et al. Protracted venous infusion 5-fluorouracil with concomitant radiotherapy compared with bolus 5-fluorouracil for unresectable pancreatic cancer. Am J Clin Oncol 2001; 24:155-9.

11. Safran H, Moore T, Iannitti D, et al. Paclitaxel and concurrent radiation for locally advanced pancreatic cancer. Int $J$ Radiat Oncol Biol Phys 2001; 49:1275-9.

12. Blackstock AW, Melin SA, Butler JM, et al. Irinotecan/gemcitabine followed by twice-weekly gemcitabine/radiation in locally advanced pancreatic cancer. Oncology (Huntingt) 2002; 16(Suppl 5):25-8.

13. Crane $\mathrm{CH}$, Abbruzzese JL, Evans DB, et al. Is the therapeutic index better with gemcitabine-based chemoradiation than with 5 -fluorouracil-based chemoradiation in locally advanced pancreatic cancer? Int J Radiat Oncol Biol Phys 2002; 52:1293-302.

14. Blackstock AW, Tepper JE, Niedwiecki D, Hollis DR, Mayer RJ, Tempero MA. Cancer and leukemia group B (CALGB) 89805: phase II chemoradiation trial using gemcitabine in patients with locoregional adenocarcinoma of the pancreas. Int $J$ Gastrointest Cancer 2003; 34:107-16.
15. Aristu J, Canon R, Pardo F, et al. Surgical resection after preoperative chemoradiotherapy benefits selected patients with unresectable pancreatic cancer. Am J Clin Oncol 2003; 26:30-6.

16. Rich T, Harris J, Abrams R, et al. Phase II study of external irradiation and weekly paclitaxel for nonmetastatic, unresectable pancreatic cancer: RTOG-98-12. Am J Clin Oncol 2004; 27:51-6.

17. Chung HW, Bang SM, Park SW, et al. A prospective randomized study of gemcitabine with doxifluridine versus paclitaxel with doxifluridine in concurrent chemoradiotherapy for locally advanced pancreatic cancer. Int J Radiat Oncol Biol Phys 2004; 60:1494-1501.

18. Wilkowski R, Thoma M, Schauer R, Wagner A, Heinemann V. Effect of chemoradiotherapy with gemcitabine and cisplatin on locoregional control in patients with primary inoperable pancreatic cancer. World $J$ Surg 2004; 28:1011-8.

19. Magnino A, Gatti M, Massucco P, et al. Phase II trial of primary radiation therapy and concurrent chemotherapy for patients with locally advanced pancreatic cancer. Oncology 2005; 68:493-9.

20. Capussotti L, Massucco P, Ribero D, Viganò L, Muratore A, Calgaro M. Extended lymphadenectomy and vein resection for pancreatic head cancer. Outcomes and implications for therapy. Arch Surg 2003; 138:1316-22.

21. Sobin LH, Wittekind Ch, eds. UICC TNM Classification of Malignant Tumours, 6th ed. New York: John Wiley \& Sons, 2002.

22. Kim HJ, Czischke K, Brennan MF, Conlon KC. Does neoadjuvant chemoradiation downstage locally advanced pancreatic cancer? J Gastrointest Surg 2002; 6:763-9.

23. Wanebo HJ, Glicksman AS, Vezeridis MP, et al. Preoperative chemotherapy, radiotherapy, and surgical resection of locally advanced pancreatic cancer. Arch Surg 2000; 135:81-8.

24. Fuhrman GM, Charnsangavej C, Abruzzese JL, et al. Thinsection contrast-enhanced computed tomography accurately predicts the resectability of malignant pancreatic neoplasms. Am J Surg 1994; 167:104-11.

25. Freeny PC, Traverso LW, Ryan JA. Diagnosis and staging of pancreatic adenocarcinoma with dynamic computed tomography. Am J Surg 1993; 165:600-6.

26. Tseng JF, Raut CP, Lee JE, et al. Pancreaticoduodenectomy with vascular resection: margin status and survival duration. $J$ Gastrointest Surg 2004; 8:935-50.

27. Breslin TM, Hess KR, Harbison DB, et al. Neoadjuvant chemoradiotherapy for adenocarcinoma of the pancreas: treatment variables and survival duration. Ann Surg Oncol 2001; $8: 123-32$.

28. Moutardier V, Magnin V, Turrini O, et al. Assessment of pathologic response after preoperative chemoradiotherapy and surgery in pancreatic adenocarcinoma. Int J Radiat Oncol Biol Phys 2004; 60:437-43.

29. White RR, Xie HB, Gottfried MR, et al. Significance of histological response to preoperative chemoradiotherapy for pancreatic cancer. Ann Surg Oncol 2005; 12:214-21.

30. Pingpank JF, Hoffman JP, Ross EA, et al. Effect of preoperative chemoradiotherapy on surgical margin status of resected adenocarcinoma of the head of the pancreas. $J$ Gastrointest Surg 2001; 5:121-30.

31. White RR, Paulson EK, Freed KS, et al. Staging of pancreatic cancer before and after neoadjuvant chemoradiation. J Gastrointest Surg 2001; 5:626-33.

32. Spitz FR, Abbruzzese JL, Lee JE, et al. Preoperative and postoperative chemoradiation strategies in patients treated with pancreaticoduodenectomy for adenocarcinoma of the pancreas. J Clin Oncol 1997; 15:928-37.

33. Snady H, Bruckner H, Cooperman A, Paradiso J, Kiefer L. Survival advantage of combined chemoradiotherapy compared with resection as the initial treatment of patients with regional pancreatic carcinoma. An outcomes trial. Cancer 2000; $89: 314-27$. 